\title{
External Thrust Induced by High-Pressure Pulse Detonation System
}

\author{
Hsin-Po Chen ${ }^{1, a}$, Chia-Nan Wang ${ }^{1, b}$ and Ming-Hsien Hsueh ${ }^{1, c}$ \\ ${ }^{1}$ Department of Industrial Engineering and Management,National Kaohsiung University of Applied \\ Sciences, Kaohsiung, Taiwan; \\ ahsinpo2@gmail.com, ${ }^{\mathrm{b}}$ cn.wang@cc.kuas.edu.tw, ${ }^{\mathrm{c}}$ mhhsueh@kuas.edu.tw
}

Keywords: Pulse detonation engine, compression ratio, detonation velocity.

\begin{abstract}
This study investigated the reaction characteristics in a pulse detonation engine, including pressure, temperature, compression ratio, sonic velocity, and detonation velocity, using CEC code and STAR-CD. The former was used for the calculation of equilibrium states, whereas the latter was used for the simulation of reaction dynamics. A complete pulse detonation tube and measurement system were designed for these experiments with monitoring and recording performed using Lab VIEW. The ignition energy as well as detonation pressure and temperature were studied experimentally and the results were compared with numerical predictions. Our results provide useful insight into the design and performance of pulse detonation engines.
\end{abstract}

\section{Introduction}

Hoffmann [1] was the first to study intermittent detonative combustion using long, small-caliber aluminum alloy tubes filled with acetylene-oxygen and benzene-oxygen fuel mixtures. Experiment results led to two main conclusions: (1) The length of the tube and the location of ignition within the tube are strongly associated with the propagation of the mixed reactants; (2) Limitations in the injection frequency of the system narrow the ignition frequency range available for the creation of intermittent and periodic supersonic combustion waves. The amount of energy that a spark plug ignition system is able to release is very limited and the highest periodic frequency in intermittent supersonic combustion is determined by the time of deflagration-to-detonation transition (DDT). Shortening the DDT process can lead to a significant decrease in the cycle period. Obtaining high thrust from supersonic combustion engines requires that they be operated at high frequencies. This is largely determined by DDT length, which is associated with the amount of energy released by the ignition system, the frequency of mixed reactant injection, and the frequency of ignition.

In recent years, many studies have found that the thrust created in the detonation system of PDEs (i.e., those that produce thrust using supersonic combustion), display Humphrey cycles at constant volume during the heat cycle. These methods are more efficient than the constant-pressure Brayton cycles found in conventional engines. Moreover, they are simple in structure and cost less to manufacture and maintain. PDEs also offer potentially higher performance, thanks to the fact that the rapid detonation process involves constant-volume combustion, which leads to higher compression ratios (between 17 and 35 using mixtures of air and propane). This is far higher than that of conventional turbine engines. It is expected that researchers will be able to expand the applicability of these methods beyond use as a substitute driving force in aircraft. These methods could theoretically include anything that requires thrust, such as electric/pneumatic hand tools and generators. Notions concerning the application of PDEs have switched to the use of outward thrust from high-pressure detonations and researchers are increasingly seeking to alter the location of moving or stationary materials in order to expand the scope of applicability. Systems with outward thrust derived from high-pressure detonations can alter the direction in which moving or stationary materials move. The most common example is when a vehicle gets a blowout on the highway, and the resulting high-pressure shockwave is sufficient to shake other vehicles nearby. If we could make use of the force from high-pressure detonations, the possibilities would be endless. 


\section{Methodology}

This study compared data from numerical simulations and actual experiments. The research instruments included the NASA CEC (Chemical Equilibrium Compositions) code [2] and STAR-CD. CEC code is used to explore the equilibrium within detonation tubes from the perspective of thermodynamics as well as the changes in detonation pressure, detonation temperature, sonic velocity, compression ratio, temperature ratio, and detonation velocity. STAR-CD simulation is used to reveal the non-steady states of pressure, temperature, and detonation velocity in detonation tubes. This study created supersonic detonation waves within a detonation tube on a test platform, the results of which were monitored using LabVIEW software. The pressure of the shockwaves was measured to explore the influence of combustion gases on detonation and identify the range of combustion gas ratios that could produce a detonation and how and where the detonation would occur. A comparison between simulation and experiment results could provide a valuable reference for the future design of PDEs.

CEC code: In this study, the theoretical mixing ratio refers to the mixing ratio of oxygen and propane:

$\mathrm{C}_{3} \mathrm{H}_{8}+5 \mathrm{O}_{2} \rightarrow 3 \mathrm{CO}_{2}+4 \mathrm{H}_{2} \mathrm{O}$

With a ratio of approximately 5:1 (under normal work temperature and operational conditions), propane and oxygen can theoretically turn into carbon dioxide and water with no additional bi-products. We thus adopted an oxygen/propane mixing ratio of $5: 1$ as our central value and performed simulations using ratios of $1: 1,2: 1,3: 1,4: 1,5: 1,6: 1,7: 1,8: 1,9: 1$, and 10:1 to identify the most efficient ratio, which would then be used to verify the results of actual testing.

With regard to initial pressure, simulations were conducted at $1 \mathrm{~atm}$ and $2 \mathrm{~atm}$. The objective was to perform two situations in the test chamber. In the first, the chamber was initially under high vacuum. The injection of reactants was performed in a controlled and precise manner according to their molar ratios until atmospheric pressure was reached. In the second situation, the chamber was initially set at atmospheric pressure and reactants were injected until the chamber reached 2 atm.

STAR-CD: Reactions within detonation tubes involve mass transfer, hydromechanics, heat conduction and combustion; therefore the governing equations include the conservation of mass, momentum, energy, turbulence energy, and turbulence dissipation rate, as shown below.

Conservation of mass continuity:

$\frac{1}{\sqrt{g}} \frac{\partial}{\partial t}(\sqrt{g} \rho)+\frac{\partial}{\partial x_{j}}\left(\rho \tilde{u}_{j}\right)=s_{m}$

Conservation of momentum:

$\frac{1}{\sqrt{g}} \frac{\partial}{\partial t}\left(\sqrt{g} \rho u_{i}\right)+\frac{\partial}{\partial x_{j}}\left(\rho \tilde{u}_{j} u_{i}-\tau_{i j}\right)=-\frac{\partial p}{\partial x_{i}}+s_{i}$

The equation for the conservation of energy (static entropy) can be expressed as follows:

$\frac{1}{\sqrt{g}} \frac{\partial}{\partial t}(\sqrt{g} \rho h)+\frac{\partial}{\partial x_{j}}\left(\rho \bar{u}_{j} h-F_{h, j}\right)=\frac{1}{\sqrt{g}} \frac{\partial}{\partial t}(\sqrt{g} p)+u_{j} \frac{\partial p}{\partial x_{j}}+\tau_{i j} \frac{\partial u_{i}}{\partial x_{j}}+s_{h}$

where $\mathrm{h}$ (static enthalpy) is defined as

$h=\overline{c_{p}} T-c_{p}^{0} T_{0}+\sum m_{m} H_{m}=h_{t}+\sum m_{m} H_{m}$

Conservation of turbulence energy $(\mathrm{K})$ :

$\frac{1}{\sqrt{g}} \frac{\partial}{\partial t}(\sqrt{g} \rho K)+\frac{\partial}{\partial x_{j}}\left(\rho \bar{u}_{j} K-\frac{\mu_{e f f}}{\sigma_{k}} \frac{\partial K}{\partial x_{j}}\right)=\mu_{t}\left(P+P_{B}\right)-\rho \varepsilon-\frac{2}{3}\left(\mu_{t} \frac{\partial u_{i}}{\partial x_{i}}+\rho K\right) \frac{\partial u_{i}}{\partial x_{i}}$

Conservation of turbulence dissipation rate $(\varepsilon)$ :

$$
\frac{1}{\sqrt{g}} \frac{\partial}{\partial t}(\sqrt{g} \rho \varepsilon)+\frac{\partial}{\partial x_{j}}\left(\rho \bar{u}_{j} \varepsilon-\frac{\mu_{e f f}}{\sigma_{\varepsilon}} \frac{\partial \varepsilon}{\partial x_{j}}\right)=C_{\varepsilon 1} \frac{\varepsilon}{K}\left[\mu_{t}\left(P+C_{\varepsilon 3} P_{B}\right)-\frac{2}{3}\left(\mu_{t} \frac{\partial u_{i}}{\partial x_{i}}+\rho K\right) \frac{\partial u_{i}}{\partial x_{i}}\right]-C_{\varepsilon 2 \rho} \rho \frac{\varepsilon^{2}}{K}-C_{\varepsilon 4} \rho \varepsilon \frac{\partial u_{i}}{\partial x_{i}}
$$

Geometric shape and mesh distribution: Fig.1 displays the geometric shape and mesh distribution in the detonation tube. Uneven distribution was adopted to obtain more reasonable results and an axisymmetric model was used to simplify the calculations. Table 1 illustrates the influence of mesh grid size in different directions on exit velocity, to which we referred in order to ensure that the pressure and velocity fields in the detonation tube were not affected by the number of grid squares in the mesh. As can be seen, the difference in velocity between $\mathrm{dx}=0.508$ and $\mathrm{dx}=0.254$ is only $0.94 \%$; 
therefore, we adopted 0.508 in all cases in this study. The resulting mesh contained a total of 21,664 grid squares.

Table 1 Influence of mesh size in different directions on exit velocity

\begin{tabular}{|l|l|l|l|l|}
\hline $\mathrm{dx}(\mathrm{mm})$ & 1.905 & 1.016 & 0.508 & 0.254 \\
\hline $\mathrm{Vd}(\mathrm{m} / \mathrm{s})$ & 822 & 810 & 803 & 795.5 \\
\hline Error & $3.33 \%$ & $1.8 \%$ & $0.94 \%$ & - \\
\hline
\end{tabular}
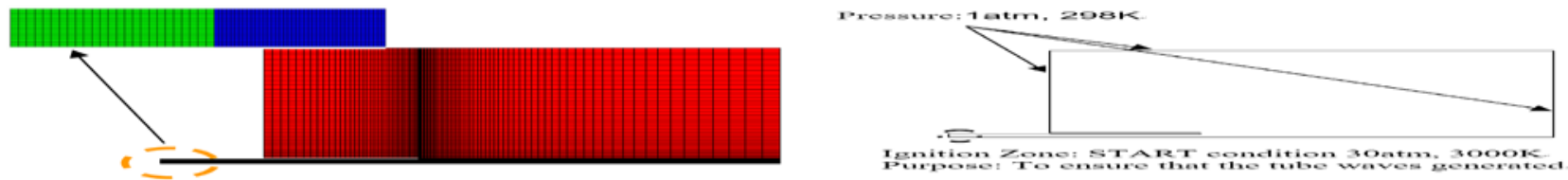

Fig. 1 Geometric shape and mesh distribution \& Inside detonation tube and initial conditions

Boundary conditions and initial conditions: Two dimensions were used for the analysis of boundary conditions: a periodic boundary and a pressure boundary of 1 atm at the exit, at a temperature of $25^{\circ} \mathrm{C}$. In regions other than the ignition area within the detonation tube, the pressure was $2 \mathrm{~atm}$, and the temperature was $25{ }^{\circ} \mathrm{C}$. To ensure the generation of shockwaves, the initial conditions of the ignition area were set at $30 \mathrm{~atm}$ and $3000 \mathrm{~K}$. Fig.1 displays the boundary conditions inside the detonation tube and the initial conditions of the ignition area. With regard to the progress of time, we adopted the monotone advection and reconstruction scheme (MARS), which is a multidimensional second-order accurate differencing scheme, to calculate non-steady state processes with precision.

\section{Empirical analysis}

\section{CEC equilibrium:}

We first used CEC code to render simulations aimed at determining the degree to which fuel quantity and proportions influence the detonation wave. This study adopted propane (C3H8) as fuel and air as the oxidizing agent in the form of air in which nitrogen accounted for $78.08 \%$ and oxygen accounted for $20.95 \%$ of the total volume. Our results include compression ratio, detonation pressure, temperature ratio, detonation temperature, sonic velocity, and detonation velocity.

Compression ratio: Compression ratio refers to the pressure ratio in the combustion mixture before and after detonation (detonation pressure/initial pressure). Fig.3 illustrates the relationship between the equivalence ratio $\left({ }^{\phi}\right)$ and the compression ratio in mixtures with different initial pressures. The results present nearly Gaussian distribution with the two ends of the curves gradually rising upwards and the peak at approximately $\phi=1.25\left(\mathrm{P}_{2} / \mathrm{P}_{1}=18.9-19.6\right.$, depending on the initial pressure). With the mixture of propane and air used in this study, the compression ratio ranged from 11.5 to 19.6. Fig.3 shows that when the mixture is in the fuel-lean range $\left(\phi_{<1)}\right.$, the compression ratio is extremely sensitive to ${ }^{\phi}$. In contrast, within the fuel-rich range, particularly when $\phi_{>3}$, the influence of $\phi$ on the compression ratio is less apparent. Furthermore, differences in initial pressure were also shown to vary with regard to their influence on compression ratio. With the same $\phi$, increasing the initial pressure does not increase the compression ratio. When $\phi=1.25$ and the initial pressure is increased from $1 \mathrm{~atm}$ to $5 \mathrm{~atm}$, the compression ratio rises by less than $1 \%$. When $\phi=5$ and the initial pressure is increased from $1 \mathrm{~atm}$ to $5 \mathrm{~atm}$, the compression ratio rises by less than $3.4 \%$.

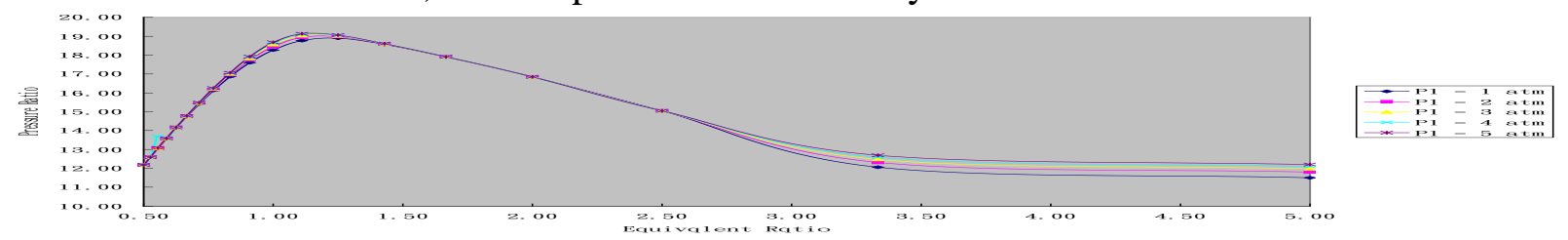

Fig.3 Pressure ratio \& equivalence ratio distribution 
Detonation pressure : Despite the fact that increasing the initial pressure does not significantly increase the compression ratio, it exerts additional influence on the pressure created by the detonation wave following ignition of the mixture. Respectively display the influence of different initial pressures and equivalence ratios on detonation pressure. With the initial pressure as the horizontal axis and the detonation pressure as the vertical axis, the former shows that initial pressure is linearly proportional to detonation pressure, regardless of mixture. With an initial pressure of $1 \mathrm{~atm}$ and an equivalence ratio of 1.0 , the detonation pressure could reach $18.3 \mathrm{~atm}$. However, when the initial pressure was increased the $2 \mathrm{~atm}$, the detonation pressure could reach $36.9 \mathrm{~atm}$ with the same mixture. The results in Fig. 4 show that the slope is steepest when $\phi=1.11$. In other words, with this equivalence ratio, increasing the initial pressure increases the detonation pressure to a greater degree.

Exhibits the relationship between the equivalence ratio and detonation pressure. A comparison of Figs. 3 and 4 shows that the maximum detonation pressure occurs between $\phi=1$ and $\phi=1.5$, regardless of the initial pressure. In the mixture with an initial pressure of $1 \mathrm{~atm}$, for instance, the detonation pressure ranges between $11.5 \mathrm{~atm}$ and $18.9 \mathrm{~atm}$. At the same time, when $\phi$ is closer to $1-1.5$, the increase in pressure is more pronounced. When the initial pressure of the fuel in the detonation tube increases, the pressure of the resulting detonation wave increases as well.
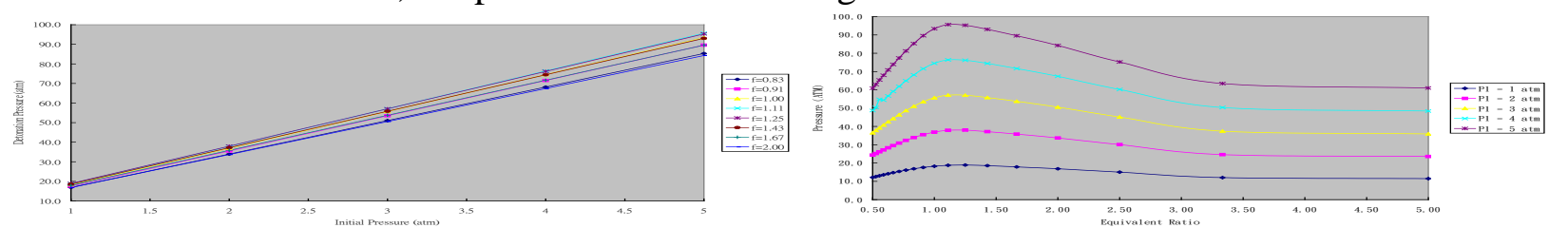

Fig.4 Detonation pressure and initial pressure distribute \& Detonation pressure and fuel ratio distribution

Temperature ratio and detonation temperature :Fig.5 illustrates the relationship between the equivalence ratio and the temperature ratio. Similar to the Fig.3, these results show a nearly Gaussian distribution. The peaks also occur when $\phi$ is between 1 and 1.5. The highest temperature ratio appeared when the initial pressure was 5 atm and $\phi=1.11$.

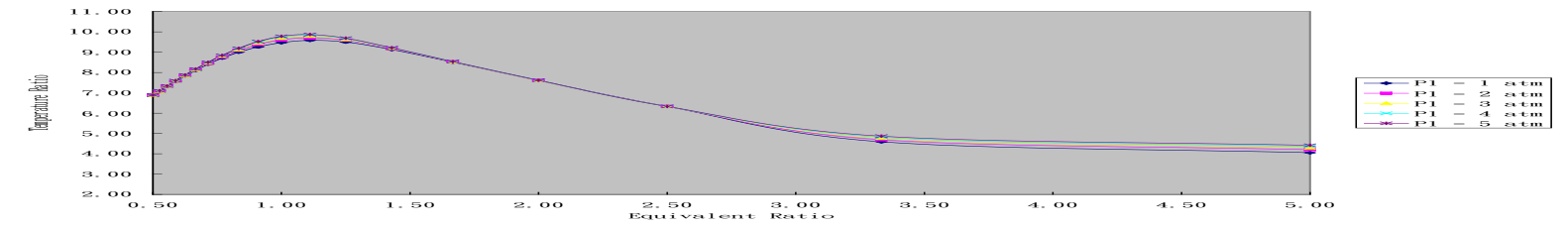

Fig.5 Temperature ratio and equivalence ratio distribution

Detonation temperature : The responses of detonation temperature are similar to those of detonation pressure. Fig.6 respectively display the influence of initial pressure and $\phi$ values on detonation temperature. The former exhibits almost parallel lines for detonation temperature with regard to initial pressure. The detonation temperature ranges from 1200 to $2900 \mathrm{~K}$, with $\phi=1.11$ resulting in the highest temperatures and $\phi=12.0$ leading to the lowest. Despite the fact that increasing the initial temperature increases the detonation temperature, the effect is almost nonexistent. Illustrates the relationship between equivalence ratio and detonation temperature. Again, the results show nearly Gaussian distribution, in which the detonation temperature rises as the equivalence ratio progresses from the fuel-lean range to the fuel-rich range. The temperature peaks at $\phi=1.11$, beyond which the detonation temperature decreases as the equivalence ratio increases. Also shows that when $\phi>3$, the variations in detonation temperature are less apparent than those when $\phi<3$. 

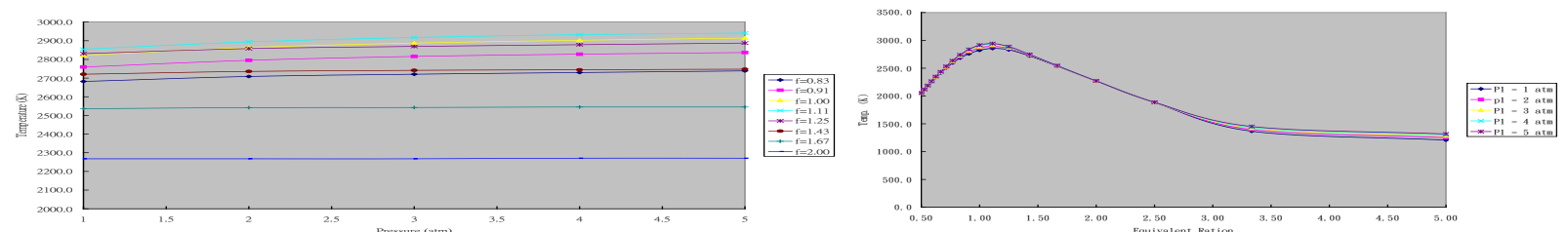

Fig.6 Detonation temperature and initial pressure distribution \& Detonation temperature and fuel ratio distribution

Sonic velocity : CEC code simulation was used to illustrate sonic velocity, which is the velocity of sound in combustion gasses following detonation. Fig.7 presents the relationship between the equivalence ratio and sonic velocity in mixtures under various initial pressures. The results are similar to those for the temperature ratio, because the environmental temperature is a factor of sonic velocity. Thus, differences in fuel pressure in the detonation tube produced sonic velocities from 788 $\mathrm{m} / \mathrm{sec}$ to $1040 \mathrm{~m} / \mathrm{sec}$ in the detonation waves. However, increasing the ratio of propane and decreasing the ratio of air led to a gradual decline in the velocity of sound.

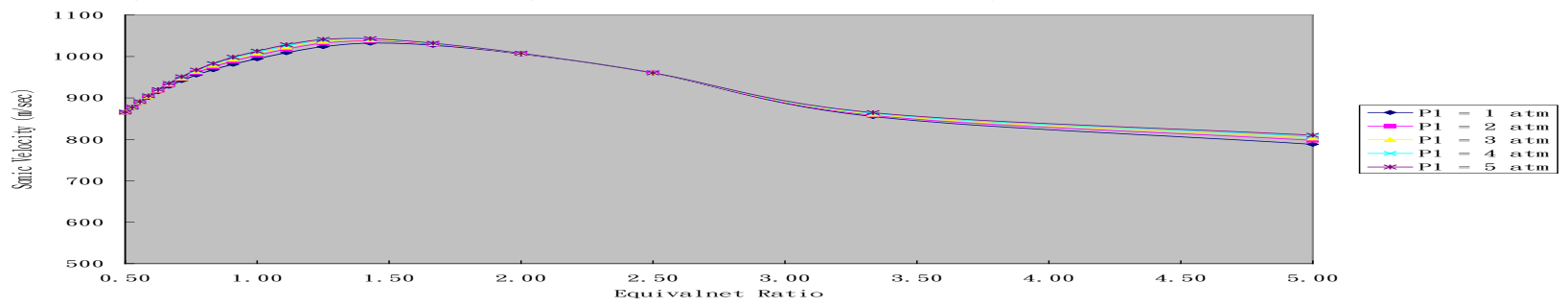

Fig.7 Sonic velocity and fuel equivalence ratio distribution

Detonation velocity : Detonation velocity and detonation pressure are two standards used to determine whether a mixture can achieve detonation. Fig.8 illustrates the relationship between the equivalence ratio and detonation velocity, with curves similar to those for compression ratio and temperature ratio. Illustrates the relationship between initial pressure and detonation velocity, which present parallel curves with some crossovers. Show that the highest detonation velocity of 1855.6 $\mathrm{m} / \mathrm{sec}$ occurred when $\phi$ was under an initial pressure of 1.25 or $5 \mathrm{~atm}$. In contrast, the lowest detonation velocity of $1384.8 \mathrm{~m} / \mathrm{sec}$ occurred when $\phi$ was under an initial pressure of 5 or $1 \mathrm{~atm}$. In other words, the detonation velocity is highest when $\phi$ is between 1.0 and 1.5. Detonation pressure declines as $\phi$ increases or decreases, and detonation velocity is higher when the initial pressure is higher.
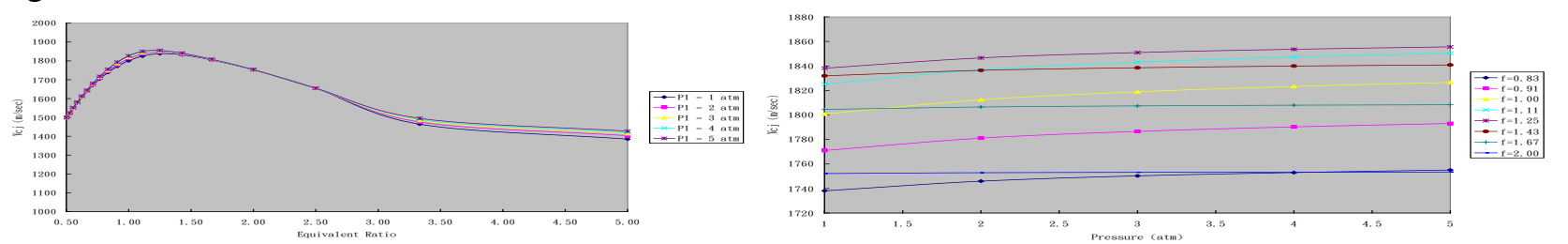

Fig.8 Detonation velocity and equivalence ratio distribution\& Detonation velocity and initial pressure distribution

\section{STAR-CD calculation results}

Fig.9 illustrates the influence of equivalence ratio on pressure in the ignition area. Simulation results reveal that detonation began at $10 \sim 15 \mu \mathrm{s}$, whereupon the pressure increased rapidly. In other words, fuel-rich conditions create better detonations than fuel-lean conditions. Subsequently, the pressure in the ignition area dropped sharply and stabilized at approximately $60 \mu$ s. Overall, the highest pressure ratio around the time of detonation ranged between 1.7 and 2.4.In terms of exit pressure, shows that the highest pressure was induced between $130 \mu \mathrm{s}$ and $150 \mu \mathrm{s}$, with $=1$ producing the best pressure output. If we compare the pressures before and after the change, the compression ratio was approximately 25. An understanding of this fact could help to improve the design of detonation tubes in the future. Illustrates the influence of equivalence ratio on exit velocity. 
Maximum pressure and exit velocity occurred at nearly the same time. The difference is that while exit velocity decreased with time, the decline is not substantial; exit velocity even rose a little between $250 \mu \mathrm{s}$ and $300 \mu \mathrm{s}$. Overall, the maximum exit velocities were obtained between $1000 \mathrm{~m} / \mathrm{s}$ and $1100 \mathrm{~m} / \mathrm{s}$. Within the time range of the simulations, the velocities remained around $800 \mathrm{~m} / \mathrm{s}$, even after decreasing.

Fig.10 Illustrates the influence of detonation tube diameter on pressure within the ignition area when the equivalence ratio equals 1 . As can be seen, the variations in pressure were nearly unaffected by diameter. It may be that the ignition area was too small for the tube diameter to exert any influence on pressure. Despite the fact that tube diameter had little impact on pressure in the ignition area, Indicates that tube diameter wielded influence on exit pressure. Overall, a larger diameter resulted in greater exit pressure. This may be attributed to the fact that a detonation tube with larger volume provided the fuel with more room for reaction, thereby making the effects of gas expansion more pronounced, which subsequently increased the exit pressure. After $400 \mu \mathrm{s}$, the influence of tube diameter on exit pressure became extremely small. Illustrates the influence of detonation tube diameter on exit velocity. Unlike the results for exit pressure, exit velocity increased with a decrease in tube diameter.
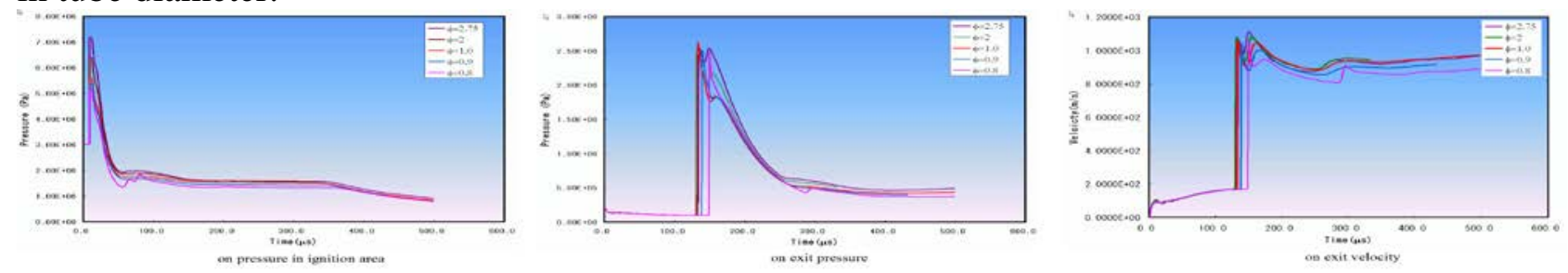

Fig. 9 Influence of equivalence ratio

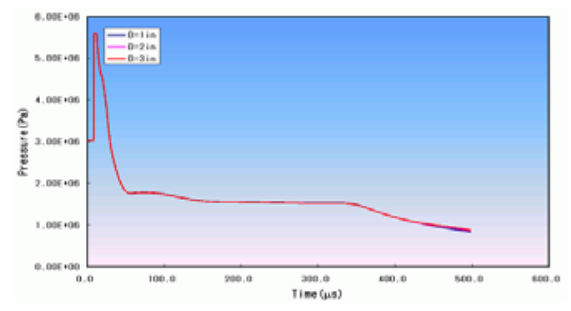

on pressure in ignition area $(=1)$

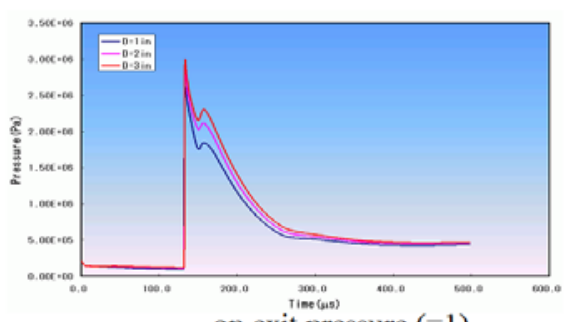

on exit pressure $(=1)$

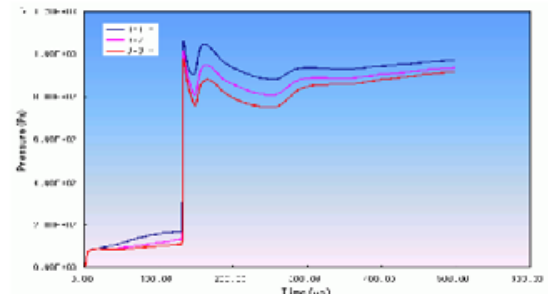

on exit velocity $(=1)$

Fig. 10 Influence of detonation tube diameter

\section{Conclusion}

Our CEC code simulation results show that the highest compression ratio $\left(\mathrm{P}_{2} / \mathrm{P}_{1}\right)$ is achieved when $\phi$ is approximately 1.25 with initial pressure ranging from 18.9 to 19.6 . When the mixture was in the fuel-lean range ( $\phi_{<1}$ ), the compression ratio was extremely sensitive to $\phi$. In contrast, within the fuel-rich range $\left(\phi_{<1}\right)$, particularly when $\phi_{>3}$, the influence of $\phi$ on the compression ratio is less apparent. According to $\phi$ values, the detonation temperature ranged from $1200 \mathrm{~K}$ to $2900 \mathrm{~K}$, with $\phi=1.11$ resulting in the highest temperatures and $\phi=2.0$ leading to the lowest. The STAR-CD simulation results indicate that detonation began at $10 \sim 15 \mu \mathrm{s}$, whereupon the pressure increased swiftly. Furthermore, higher $\phi$ values had a more pronounced impact on the maximum pressure in the ignition area. In other words, fuel-rich conditions created better detonations than fuel-lean conditions. The maximum exit velocities ranged from $1000 \mathrm{~m} / \mathrm{s}$ to $1100 \mathrm{~m} / \mathrm{s}$. This study obtained a basic understanding of the reaction characteristics in PDEs. In future research, we will continue to make progress towards intermittent ignition with the aim of creating supersonic detonation waves with a detonation tube and test platform, monitored using LabVIEW. The pressure of the shockwaves will be measured to explore the influence of combustion gases on detonation and identify the range of combustion gas ratios capable of producing detonations and determining how and where the 
detonations occur. The comparison between the simulation and experiment results provides a valuable reference for the future design of PDEs.

\section{References}

[1] Hoffmann, N.,"Reaction Propulsion by Intermittent Detonative Combustion,” Ministry of Supply, Volkenrode Translation, 1940.

[2] Gordon, S. and McBride, B. J., “computer Program for Calculation of Complex Chemical Equilibrium Compositions, Rocket Performance, Incident and Reflected Shocks, and Chapman-Jouguet Detonation,” NASA SP 273, 1971. 